\title{
Chapter 17 \\ Es Tiempo: Engaging Latinas in Cervical Cancer Research
}

\author{
Lourdes Baezconde-Garbanati, Carol Y. Ochoa, Sheila T. Murphy, \\ Megan B. Moran, Yaneth L. Rodriguez, Rosa Barahona, and Lizette Garcia
}

\section{Overview of Community-Driven Research}

Community health science is an approach that takes science to the community and ensures that the community participates in the scientific process in order to improve quality of life [1]. It is the development of a two-way street between communities and academia that helps solve some of the most challenging scientific health problems at the community level. Academic-community partnerships provide one mechanism to facilitate engagement of communities in research. The goal is to improve access to services, research participation, access to technology, and information/knowledge transfer or faster dissemination of information. As the Latino community ages, grows, and spreads throughout the United States, bridging the gap between science and practice becomes more urgent, especially among older segments of the Latino population whose cancer risk profile may be increasing. This is needed especially in cancer prevention and control, if Latinos are to benefit from new and emerging technological advances and engage in precision medicine more fully.

\footnotetext{
L. Baezconde-Garbanati $(\varangle) \cdot$ Y. L. Rodriguez $\cdot$ R. Barahona $\cdot$ L. Garcia Institute for Health Promotion and Disease Prevention Research, University of Southern California, Los Angeles, CA, USA

e-mail: Baezcond@usc.edu

C. Y. Ochoa

Department of Preventive Medicine, Keck School of Medicine, University of Southern California, Los Angeles, CA, USA

S. T. Murphy

Annenberg School for Communication and Journalism, University of Southern California, Los Angeles, CA, USA

M. B. Moran

Department of Health, Behavior and Society, Johns Hopkins University,

Baltimore, MD, USA
} 
In the United States, Latino communities are changing demographically and culturally. Latinos are not a monolithic unit, but are instead a very heterogeneous group with differences in acculturation, immigration, documentation status, country of origin, and racial/ethnic make-up. In spite of this heterogeneity, their connection with each other is central to the Latino community. The values that Latinos share are central to the essence and culture of being Latino. Most share in the Spanish language, except Brazilians who speak Portuguese and some indigenous communities from Central and South America who predominantly speak an indigenous language, even if they have learned Spanish. Common to the culture is an emphasis on personalismo, familismo, simpatia, collectivism, harmony, and cooperation in the group $[2,3]$. These characteristics have often been found to be protective and have served as deterrents to unhealthy life styles, such as alcohol, drug, and tobacco use in some segments of the Latino community. Although other groups may share in similar values, solutions found for other cultural/ethnic and racial groups may not necessarily apply to Latinos and to Latinas in particular. For example, the Latino Epidemiologic Paradox shows that in spite of living in poverty and having low educational attainment, some segments of the Latino population have very positive health outcomes on specific indicators and have shown important gains in life expectancy.

Unfortunately, Latino populations are not always well informed of recent advances in research, nor do they benefit equally from scientific discoveries as other populations. On average, research can take 17 years to go from bench to bedside [4], which is why community-based models are needed; these models engage participants in research, not just as subjects but also as planners, implementers, and disseminators of information, so information can be transferred more quickly. Community-based participatory models can decrease this time gap by utilizing patient-centered approaches and by including patient advocates, citizen scientists, and community health workers, who are trusted by their respective communities to research and deliver life-saving information and accelerate knowledge transfer. These programs work best through the establishment of equal power-sharing partnerships, which effectively accelerate health improvements that more quickly achieve health equity [5]. The World Health Organization (WHO) defines equity as "the absence of avoidable or remediable differences among groups of people, whether groups are defined socially, economically, demographically, or geographically" [6]. In our research, we strive to achieve health equity in cancer-related diseases and to adequately reduce risk factors via participation in research and more timely dissemination of information to the Latino community.

Israel et al. [7] described key principles for community engagement which we utilize in our research with Latinos. These include (1) engaging community partners from the beginning stages; (2) working on the principle of shared equity and decision-making; (3) engaging in mutual learning in all stages of research; (4) negotiating a common vision, goals, values, priorities; (5) compensating community partners; (6) hiring staff from the local community; (7) training community health workers/promotoras de salud; (8) allowing community partners to influence program direction; (9) sharing equal power among partners; (10) planning jointly with 
partners; and (11) working with partners on dissemination of information. These are principles that form part of community-based participatory research (CBPR). Even though it is not always possible to implement all CBPR principles in cancer research, some cancer research can still be community driven and abide by many of the principles described above.

Our over 25 years of experience show that to actively engage Latinas in cervical cancer and other cancer-related research, it is critical to build strong relationships in the Latino community over time. We build community trust by establishing several partnerships that are still in effect years later. Further, we engage Latino communities and Latinas, in particular, directly and indirectly in the research. Latinos are engaged as participants and as community scientists. Once the research is finalized, we give information back to these communities. Further, we follow up in terms of policy or services that are needed to further community goals, and we work with volunteer organizations, such as the American Lung Association and the American Cancer Society in policy and advocacy.

One important mechanism we have utilized is the establishment of community advisory boards/committees (CABs/CACs) [8]. Through our CABs we have engaged key community members in guiding the research from its initial stages through to its dissemination [9]. This implies that the CABs are truly heard and that academic researchers recognize the importance of their contributions from conceptualization to decisions on analysis and information dissemination. We have also engaged patient advocates, community scientists, citizen scientists, and/or community health workers (CHWs) and promotores de salud as vehicles for listening to community voices. These key community representatives do not necessarily have a formal science or research background, but they contribute time, effort, and resources toward scientific research. Citizen scientists work often with professional scientists or alone [10]. Citizen scientists and patient advocates are helping to bridge gaps in research, as everyday people contribute to the scientific enterprise. In these days of Big Data, they can contribute to data collection by accumulating photographs and videos of their environment and by providing context for interpretation of data from these large datasets. Ability to help culturally interpret findings is critical.

\section{Current Work}

Although much has been accomplished in reducing morbidity and mortality from cancer, cervical cancer remains a serious health threat for Latinas in Los Angeles. In LA County, the incidence of cervical cancer is as high as 8.0 per 100,000 among Latinas, compared to 6.3 per 100,000 among Asian/Pacific Islander women, 10.5 per 100,000 among African American women, and 7.3 per 100,000 among nonHispanic White women [11]. These statistics are particularly worrisome, especially for Los Angeles, a county heavily populated by Latinos. Women present them- 
selves to local clinics with advanced stage of disease and limited survival and treatment options.

\section{Past Interventions}

A multidisciplinary team at the University of Southern California (USC) conducted 12 focus groups [12], which resulted in the development of two research/intervention programs: Tamale Lesson (Murphy/Baezconde-Garbanati, NCIR01CA144052), Es Tiempo (California Community Foundation, the Norris Comprehensive Cancer Center, P30 CA01408939S4), and the SC Clinical Translation Science Institute (CTSI-UL1TROOO130).

\section{Tamale Lesson: Transforming Cancer Information Through Narrative}

For Tamale Lesson, we developed two 11-min English films with the goal of reducing the burden of cervical cancer. Tamale Lesson was also translated and adapted into Spanish. Tamale Lesson was produced using a culturally tailored narrative format which aimed to provide information on the human papillomavirus (HPV), the virus that causes cervical cancer; cervical cancer prevention via vaccination; and cervical cancer detection via Pap test screening and new genetic tests. The second film, It's Time, was developed as a non-fictional, non-narrative alternative to Tamale Lesson and also included similar information. Details of development are presented elsewhere [13]. Short 3-min clips of these videos are available on YouTube, as well as longer versions on DVD. A sample of 900 European American, African American, and Mexican American women living in Los Angeles were recruited through random digit dialing (RDD) and were randomly assigned to receive either the narrative or the non-narrative film in the mail. All women were surveyed to assess their level of cervical cancer-related knowledge, attitudes, and behavior before receiving the film, 2 weeks and 6 months after viewing the film. Latinas participated in many aspects of the research. They were active participants in the focus groups, and also participated in focus groups that viewed and edited the films to make them more culturally specific. Through funding from the SC CTSI, promotoras de salud were included. They participated in the research via the formation of a community advisory board that produced the Spanish language version of Tamale Lesson. Findings from the study revealed that the narrative and non-narrative films were successful interventions in both the short-term at posttest and long-term at 6-month follow-up [14]. The narrative film was more effective in increasing cervical cancer-related knowledge and attitudes at posttest than the non-narrative. Moreover, the narrative film reduced cervical cancer screening disparities for Mexican American women from pretest to the 6-month follow-up. Fifty percent of non-Hispanic whites, 32\% of Mexican Americans, and 36\% of African Americans did not need a Pap test at baseline. Within 6 months after viewing the Tamale Lesson, the disparity disappeared, 
and $78 \%$ of non-Hispanic whites, $83 \%$ of Mexican Americans, and 77\% of African Americans had a Pap test or made an appointment for cancer screening [14]. The program has been disseminated not only throughout California but also across several countries in the Caribbean and Latin America, including Colombia, Mexico, Panama, Costa Rica, and Argentina through Buena Salud America of the National Alliance for Hispanic Health and the Healthy Americas Institute. In Panama, the Ministry of Health is distributing Tamale Lesson throughout 900 jurisdictions, including rural indigenous areas.

\section{Es Tiempo: The Jacaranda Initiative}

Our multidisciplinary team at USC developed Es Tiempo-a stunningly beautiful and innovative campaign to prevent cervical cancer among Latinas. It was built on the 12 Latina focus groups [13] and environmental scans conducted by students in the Designmatters Program at the ArtCenter College of Design. Es Tiempo uses the annual blooming of the purple jacaranda tree in Los Angeles to remind us all that we can prevent cervical cancer and to remind women to vaccinate their sons and daughters from HPV. Es Tiempo's design elements were created by students from the ArtCenter College of Design, Designmatters Program and developed and tested by investigators from the Norris Comprehensive Cancer Center, Institute for Health Promotion and Disease Prevention Research, Department of Preventive Medicine, Keck School of Medicine of USC, and the Annenberg School for Communication and Journalism. The program was pilot tested and launched with cooperation from community clinics, Clínicas Monseñor Oscar Romero (Pico Union and Boyle Heights). We obtained support from the Office of Women's Health (OWH) at the L. A. County Department of Public Health.

The bilingual Es Tiempo campaign has three components: (1) an outdoor media educational campaign; (2) a clinic intervention in partnership with Clínicas Monseñor Oscar A. Romero in Boyle Heights and Pico Union; and (3) a community component, where promotoras de salud deliver community educational workshops and collect intercept surveys to measure knowledge increases at the community level. Latinas are and have been engaged in this project from its creation all the way through to its implementation and dissemination in the community. Outdoor media materials include billboards, bus benches, and light post banners with the OWH cervical cancer helpline number. When Latinas call the helpline, trained callers make appointments and referrals for free or low-cost cervical cancer screening and HPV vaccinations for qualifying women. Intervention clinics mail postcards to women who have not had a Pap test in the last 3 years (based on current guidelines), and clinic banners remind women to schedule their cervical cancer screening or vaccinate their sons and daughters. OWH and the clinics report the number of women who call or make appointments pre- and post-intervention. In addition, promotoras de salud conduct community workshops which have a pre- and post-survey of knowledge on cervical cancer and the HPV vaccine. At the end of the workshop, participants are provided with referrals, places where they can receive low or no 
cost screening and HPV vaccinations. Also, women are encouraged to follow-up if they have abnormal Pap test results. Lastly, intercept community surveys are conducted to assess the campaign and understanding of the material; knowledge-related questions pertaining to cervical cancer, the HPV vaccine, and Pap test; format in which they have received health information; and how they would like to receive health information in the future.

Clínicas Monseñor Oscar Romero (community partner in Es Tiempo) reported that 1428 women were eligible for Pap tests in the community intervention clinic and 745 women were eligible from a control clinic located in another area of the city. Our findings show that there was a statistically significant difference in percent of women who became compliant during the intervention period. The percentage of women in the Boyle Heights intervention clinic was higher than the Pico Union no intervention ( $46 \%$ vs. $33 \%, p<0.01$ ). Also, there was a statistically significant difference in percent of women who received a Pap test based on the type of intervention they received. Women in the Boyle Heights clinic who received a postcard at home with the same imagery as the outdoor media had higher rates of Pap testing than women in Pico Union who received only the postcard (65\% vs. 34\%, $p<0.001)$. Our findings demonstrate the effectiveness of a multicomponent intervention to promote screening for cervical cancer that incorporates Latinas in various aspects of the program.

\section{Identified Gaps}

There continues to be a need for the inclusion of Latinas in cancer prevention and control research. Latina involvement in our research helped us develop interventions that include social and cultural assets, which are essential elements that can make a difference in the elimination of health disparities in cervical cancer screening and in HPV prevention and control. For example, our research shows that Latinas respond well to narrative or storytelling in an educational campaign such as Tamale Lesson. Feeling transported into a storyline, being able to identify with the characters, and being emotionally impacted by the narrative [14] will go a long way to help eliminate cancer disparities. There is also a need to target a more diverse population (e.g., region and age), because cultural elements that resonate best with one Latino community may not resonate best for others, such as El Paso, Chicago, Watsonville, or Miami.

\section{Future Research}

Greater engagement of Latinas in cervical cancer research may provide opportunities for eliminating this cancer health disparity. Working in partnership with Latinodriven clinics, community-based organizations, and volunteer agencies, our 
researchers can commit to the translation of research into policies and public health initiatives that have the potential for making a difference at the community level. In order to engage Latinas in cervical cancer research, we can work with the community and not only engage in research but also in its translation and dissemination, so it can reach our various communities more quickly. Es Tiempo and Tamale Lesson demonstrate ways in which we can provide a research environment that is more conducive to participation and engagement. Our campaigns and intervention programs model cultural strategies that can be used not just for cervical cancer but also for other diseases. Community-level interventions that are conceptualized, developed, and implemented using community-based participatory principles and that engage Latinas from the beginning of the research through the dissemination of data, have the potential of increasing Latina research engagement, helping to reduce cancer disparities and save lives.

Acknowledgments We wish to acknowledge NIH funding, NCI grants: P30CA014089 and R01CA144052, and NIH UL1TR000130; and the work in earlier stages of these projects of: Doe Meyer and Jeremy Kagan, USC School of Cinema, Dr. Lauren Frank, Portland University, Dr. Vickie Cortessis, Dr. Laila Muderspach, Dr. Carolina Aristazabal, Irene Martinez, Genesis Gutierrez, Sasha Blades, Marisela Robles at the Keck School of Medicine of USC, Dr. Robert Haile at Cedars Sinai Medical Center in Los Angeles, and of the Promotoras de Salud at Vision y Compromiso.

\section{References}

1. Wandersman A. Community science: bridging the gap between science and practice with community-centered models. Am J Community Psychol. 2003;31(3-4):227-42.

2. Gudykunst WB. Bridging differences: effective tntergroup communication. Thousand Oaks: SAGE Publications Inc.; 1998.

3. Marin G, Marin B. Research with Hispanic populations. Newbury Park: SAGE Publications Ltd.; 1991. https://doi.org/10.4135/9781412985734.

4. Balas EA, Boren SA. Managing clinical knowledge for health care improvement. Yearb Med Inform. 2000;1:65-70.

5. APHA. Support for community health workers to increase health access and to reduce health inequities. American Public Health Association. 2009. https://www.apha.org/policiesand-advocacy/public-health-policy-statements/policy-database/2014/07/09/14/19/supportfor-community-health-workers-to-increase-health-access-and-to-reduce-health-inequities. Accessed 19 Feb 2018.

6. WHO. Health equity. 2018. http://www.who.int/topics/health_equity/en/. Accessed 21 Feb 2018.

7. Israel BA, Schulz AJ, Parker EA, Becker AB. Review of community-based research: assessing partnership approaches to improve public health. Annu Rev Public Health. 1998;19:173-202.

8. Institute SCTS. Toolkit for developing community partnerships: CTSI, Office of Community Engagement. Los Angeles: University of Southern California; 2010.

9. Baezconde-Garbanati L, Cruz TB, Sussman S, Unger JB. Maximizing compliance with tobacco policy in vulnerable community retail environments: a multicultural case study in community-based participatory research. London: SAGE Research Methods Cases; 2017. https://doi.org/10.4135/9781526419293. 
10. SciStarter. What is citizen science? 2018. https://scistarter.com/citizenscience.html. Accessed $21 \mathrm{Feb} 2018$.

11. Los Angeles County Department of Public Health, Office of Women's Health. Health indicators for Women in Los Angeles County: highlighting Disparities by Ethnicity and Poverty Level. January 2017. Accessed at: http://publichealth.lacounty.gov/owh/docs/DataReport/2017-Healt hIndicatorsforWomeninLACounty.pdf.

12. Baezconde-Garbanati L, Murphy ST, Moran MB, Cortessis VK. Reducing the excess burden of cervical cancer among Latinas: translating science into health promotion initiatives. Calif J Health Promot. 2013;11(1):45-57.

13. Baezconde-Garbanati LA, Chatterjee JS, Frank LB, Murphy ST, Moran MB, Werth LN, et al. Tamale lesson: a case study of a narrative health communication intervention. J Commun Healthc. 2014;7(2):82-92.

14. Murphy ST, Frank LB, Chatterjee JS, Moran MB, Zhao N, Amezola de Herrera P, et al. Comparing the relative efficacy of narrative vs nonnarrative health messages in reducing health disparities using a randomized trial. Am J Public Health. 2015;105(10):2117-23.

Open Access This chapter is licensed under the terms of the Creative Commons AttributionNonCommercial 4.0 International License (http://creativecommons.org/licenses/by-nc/4.0/), which permits any noncommercial use, sharing, adaptation, distribution and reproduction in any medium or format, as long as you give appropriate credit to the original author(s) and the source, provide a link to the Creative Commons license and indicate if changes were made.

The images or other third party material in this chapter are included in the chapter's Creative Commons license, unless indicated otherwise in a credit line to the material. If material is not included in the chapter's Creative Commons license and your intended use is not permitted by statutory regulation or exceeds the permitted use, you will need to obtain permission directly from the copyright holder.

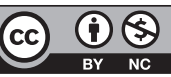

